\title{
Revisão de três espécies medicinais e ornamentais da família Apocynaceae Juss.
}

Review of three medicinal and ornamental species of the family Apocynaceae Juss.

Revisión de tres especies medicinales y ornamentales de la familia Apocynaceae Juss.

\section{Resumo}

Dentre os táxons botânicos que apresentam um alto potencial farmacológico, está a família Apocynaceae Juss.. Com isso, este trabalho teve como objetivo traçar características farmacológicas, fitoquímicas e indicações terapêuticas de 
três espécies da família supracitada. Referente à espécie Allamanda cathartica L. (dedal-de-princesa), os dados mostram que suas folhas e seu látex são utilizados para o tratamento de febre, icterícia, parasitoses, complicações da malária, baço aumentado e utilizado como laxante e purgante. Podendo tais propriedades estarem relacionadas à sua constituição fitoquímica, visto que apresenta iridóides plumericina e plumieride. Já a espécie medicinal Catharanthus roseus (L.) Don. conhecida popularmente como "boa-noite", é utilizada para o controle de hemorragias e escorbuto, como enxaguante bucal para dor de dente, na cicatrização e limpeza de feridas crônicas e no tratamento de diabetes. A espécie possui a capacidade de sintetizar uma grande variedade de compostos que são usados pelo vegetal para realizar funções biológicas, como a defesa de predadores. Os alcaloides é o grupo de compostos mais potencialmente ativo de $C$. roseus, sendo que já foram registrados mais 400 alcaloides nas diversas partes da espécie. Por fim, Nerium oleander L. (espirradeira) possui também grande potencial medicinal, com registros do seu uso no Brasil como expectorante, tônico para o coração, diurético e emético. Doses muito pequenas de extrato de folhas já foram registradas com uso em picadas de cobra. Fica evidente, que esse táxon apresenta espécies com propriedades medicinais, sendo portanto, uma fonte de compostos bioativos de interesse farmacológico.

Palavras-chave: Fitoterapia; Allamanda cathartica; Catharanthus roseus; Nerium oleander.

\begin{abstract}
Among the botanical taxa that have a high pharmacological potential is the Apocynaceae Juss family. Thus, this work aimed to trace pharmacological, phytochemical and therapeutic indications of three species of the aforementioned family. Regarding the species Allamanda cathartica L. (princess thimble), the data show that its leaves and latex are used for the treatment of fever, jaundice, parasitosis, malaria complications, enlarged spleen and used as a laxative and purgative. Such properties may be related to its phytochemical constitution, as it has plumericin and plumieride iridoids. The medicinal species Catharanthus roseus (L.) Don. popularly known as "good night", is used to control hemorrhages and scurvy, as a mouthwash for toothache, in the healing and cleaning of chronic wounds and in the treatment of diabetes. The species has the ability to synthesize a wide variety of compounds that are used by the plant to perform biological functions, such as defending against predators. The alkaloids are the most potentially active group of compounds of C. roseus, and over 400 alkaloids have been recorded in different parts of the species. Finally, Nerium oleander L. (espirradeira) also has great medicinal potential, with records of its use in Brazil as an expectorant, heart tonic, diuretic and emetic. Very small doses of leaf extract have been reported for use in snake bites. It is evident that this taxon has species with medicinal properties, being, therefore, a source of bioactive compounds of pharmacological interest.
\end{abstract}

Keywords: Phytotherapy; Allamanda cathartica; Catharanthus roseus; Nerium oleander.

\title{
Resumen
}

Entre los taxones botánicos que tienen un alto potencial farmacológico se encuentra la familia Apocynaceae Juss, por lo que este trabajo tuvo como objetivo rastrear indicaciones farmacológicas, fitoquímicas y terapéuticas de tres especies de la familia antes mencionada. En cuanto a la especie Allamanda cathartica L. (dedal princesa), los datos muestran que sus hojas y látex se utilizan para el tratamiento de fiebre, ictericia, parasitosis, complicaciones de la malaria, agrandamiento del bazo y como laxante y purgante. Tales propiedades pueden estar relacionadas con su constitución fitoquímica, ya que posee iridoides de plumericina y plumierida. La especie medicinal Catharanthus roseus (L.) Don, conocida popularmente como "buenas noches", se utiliza para controlar hemorragias y escorbuto, como enjuague bucal para el dolor de muelas, en la cicatrización y limpieza de heridas crónicas y en el tratamiento de la diabetes. La especie tiene la capacidad de sintetizar una amplia variedad de compuestos que son utilizados por la planta para realizar funciones biológicas, como defenderse de los depredadores. Los alcaloides son el grupo de compuestos más potencialmente activo de C. roseus, y se han registrado más de 400 alcaloides en diferentes partes de la especie. Finalmente, Nerium oleander L. (espirradeira) también tiene un gran potencial medicinal, con registros de su uso en Brasil como expectorante, tónico cardíaco, diurético y emético. Se han informado dosis muy pequeñas de extracto de hoja para su uso en mordeduras de serpientes. Es evidente que este taxón posee especies con propiedades medicinales, siendo, por tanto, fuente de compuestos bioactivos de interés farmacológico.

Palabras clave: Fitoterapia; Allamanda cathartica; Catharanthus roseus; Nerium oleander.

\section{Introdução}

A família Apocynaceae Juss. destaca-se por possuir espécies que são mundialmente utilizadas por comunidades para diversas finalidades, especialmente como ornamentais e medicinais (Santos et al., 2013). Compreendendo cerca de 5.000 espécies distribuídas em 400 gêneros, a família possui representantes que são amplamente utilizados na medicina tradicional (Coutinho \& Louzada, 2018), tendo em vista que essas plantas detêm substâncias biologicamente ativas, como por exemplo os alcaloides antitumorais vimblastina e vincristina de Catharanthus (Di stasi \& Hiruma-Lima, 2002).

No Brasil, são registrados 77 gêneros e 753 espécies de Apocynaceae (BFG, 2015) distribuídas nas diversas 
formações vegetacionais. Dentre os representantes desse táxon ocorrentes na região Nordeste no Brasil, as espécies Allamanda cathartica L., Catharanthus roseus (L.) Don. e Nerium oleander L. destacam-se por serem utilizadas como ornamental e para o tratamento de diversas doenças por comunidades tradicionais ao longo do território brasileiro (Santos et al., 2013).

Buscando ampliar e fixar o conhecimento relacionado as plantas brasileiras com potencial medicinal e ornamental, foi realizada uma busca na Lista da Flora do Brasil e em trabalhos acadêmicos de espécies pertencentes a Família Apocynaceae com distribuição para o Nordeste brasileiro que são utilizadas como ornamental e medicinal. Para isso, foram selecionadas três espécies de Apocynaceae (A. cathartica, C. roseus e N. oleander) que possuem distribuição para a região Nordeste do Brasil e são utilizadas como ornamental e medicinal pelas populações tradicionais (Santos et al., 2013). Posteriormente, foram reunidas informações acerca da distribuição geográfica das espécies para o Brasil, aspectos etnobotânicos e etnofarmacológicos, estudos farmacológicos, medicinais e atividades biológicas, dentro outras informações que registram a importância dessas espécies.

\section{Apocynaceae: medicinais e ornamentais}

\subsection{Allamanda cathartica $\mathbf{L}$.}

Allamanda cathartica L. (dedal-de-princesa ou dedal-de-dama) é uma espécie arbustiva nativa do Brasil e endêmica da América do Sul. Ela pode ser encontrada em todas as regiões do Brasil (Figura 1) nos domínios fitogeográficos da Amazônia, Cerrado e Mata Atlântica (Flora do Brasil, 2021).

Figura 1: Regiões de ocorrência de Allamanda catartica L. no território brasileiro.

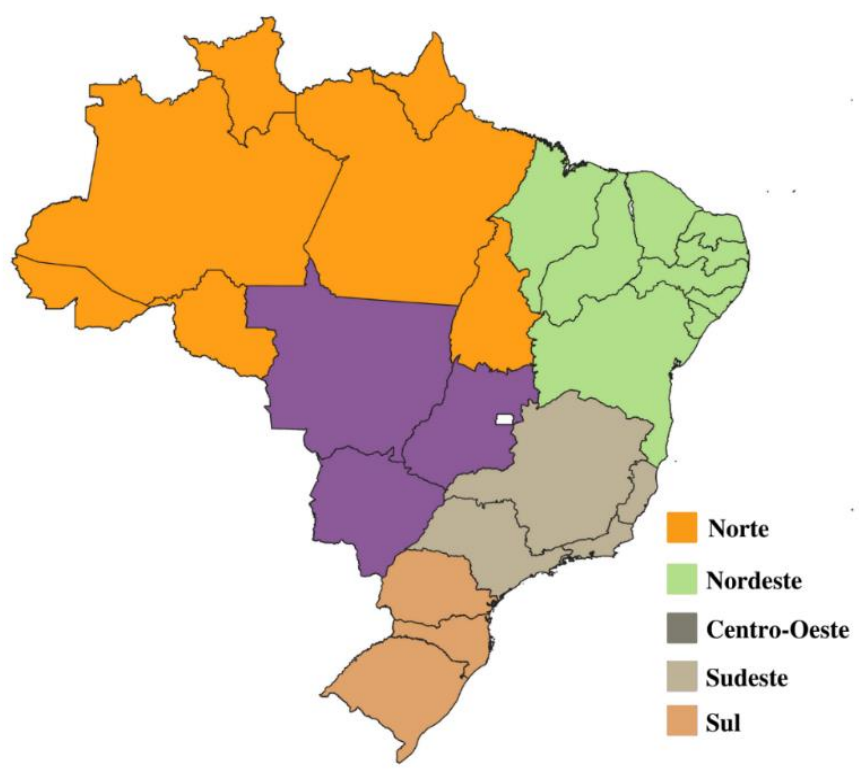

Fonte: Adaptado de Flora do Brasil (2021).

Allamanda cathartica é um arbusto que pode atingir até cerca de 6 metros de altura (Figura 2). Possui folhas elípticas a obovadas, com coloração verde escura na face adaxial e verde clara na face abaxial. Suas flores são actinomorfas com corola amarela. Possui frutos em formato de cápsulas globosas com sementes elípticas a orbiculares, aladas (Figura 2) (Petricevich \& Abarca-Vargas, 2019). 
Figura 2: Allamanda cathartica L.
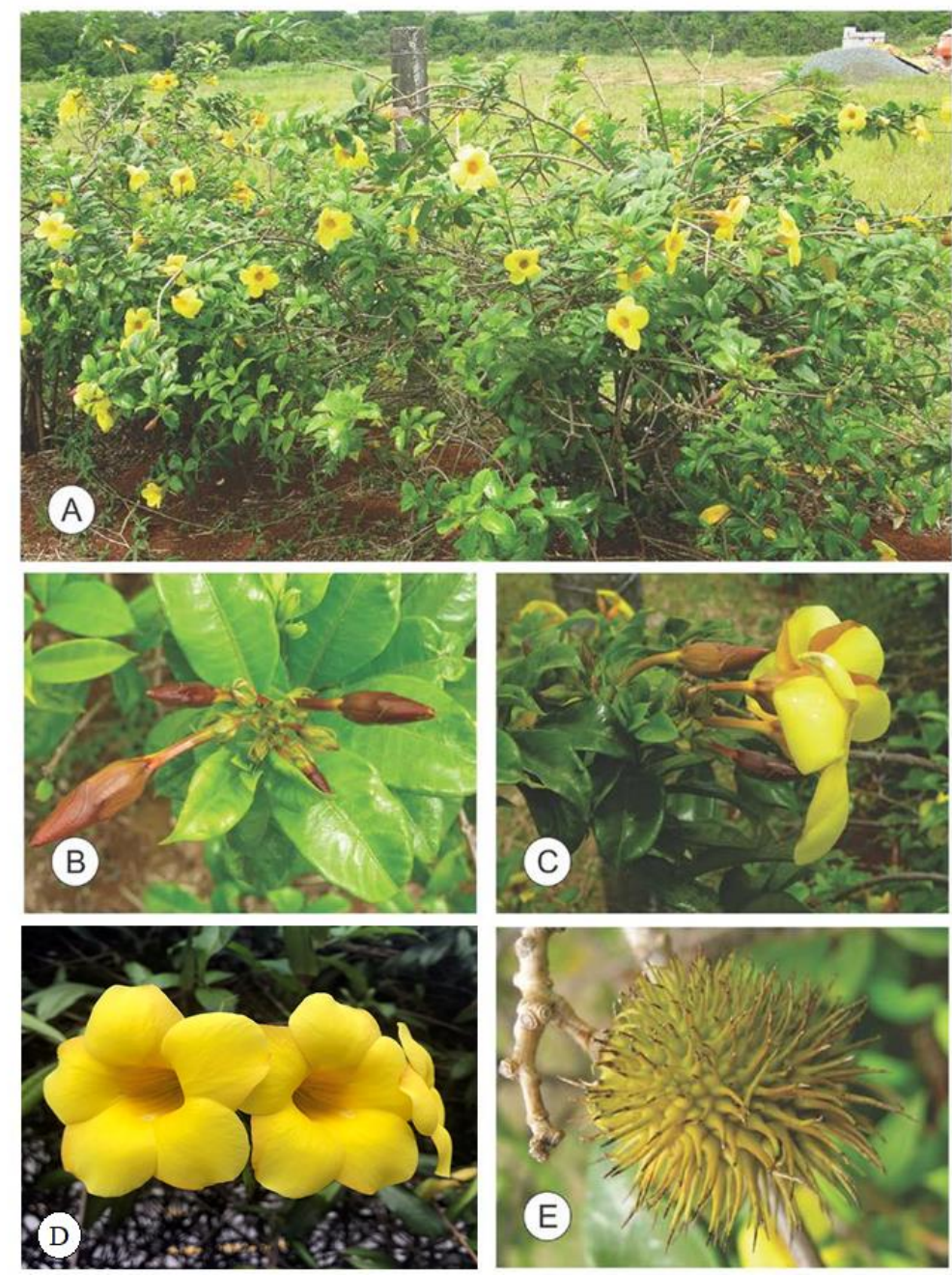

A - Hábito; B, C - Inflorescência; D - Flores e E - Fruto. Fonte: Emília R. Kotovski (2013).

No Brasil existem registros do uso das folhas e látex de A. cathartica para o tratamento de febre, icterícia, parasitoses, complicações da malária, baço aumentado e utilizado como laxante e purgante. O látex já foi usado também contra sarnas e piolhos (Scio et al., 2012; Petricevich \& Abarca-Vargas, 2019). As folhas, sob a forma de decocto, são usadas nos casos de cistites, uretrites, e no tratamento de afecções renais e como eméticas (Grandi et al., 1989). Existem registros do uso das suas cascas no tratamento de tumores hepáticos. As raízes e flores são utilizadas para intoxicação causada pelo chumbo (saturnina) (Lopes et al., 2009). As partes da planta mais utilizadas, em ordem decrescente, são as folhas, casca do caule, flores, raízes, caule, seiva, sementes e ramos (Petricevich \& Abarca-Vargas, 2019).

Na Guiana o látex é usado para cólicas, tosse e dores de cabeça (Ghosh et al., 2019). Consta, ainda, na literatura que na Colômbia, os curandeiros usam os extratos dos caules e folhas de A. cathartica para tratar os efeitos hemorrágicos do veneno de Bothrops atrox L. (Lopes et al., 2009).

São encontrados em A. cathartica os purgativos iridóides plumericina e plumieride, o primeiro nas raízes e o último em toda a planta (Burrows \& Tyrl, 2013). O iridóide alamandina foi isolado do extrato das raízes de A. catartica, e representa uma substância de grande interesse medicinal, sendo que em estudos anteriores a mesma já apresentou atividade antitumoral em células derivadas do carcinoma humano (Lopes et al., 2009). Já foram identificados também compostos pertencentes as classes dos alcaloides, antraquinonas, antocianinas, carboidratos, carotenoides, cumarina, flavonoides, glicosídeos, 
hidrocarbonetos, lignina, lipídios, compostos fenólicos, quinonas, saponinas, esteroides, taninos e terpenos em todas as partes do vegetal (Petricevich \& Abarca-Vargas, 2019).

Já foram registradas atividades analgésica, antidepressiva, cicatrizante, trombolítica, purgativa, antiviral, antioxidante, antimalárica e antidiabético das folhas A. cathartica. A espécie apresentou também efeito anti-inflamatório (flores), nematicida (cascas) e pesticida (folhas e flores) (Petricevich \& Abarca-Vargas, 2019). Mostrou importante atividade in vitro contra o carcinoma humano da nasofaringe. Os extratos das folhas apresentaram importante efeito antibacteriano contra organismos patogênicos, sendo estes, Escherichia coli, Pseudomonas aeruginosa, Klebsiella pneumoniae e Proteus mirabilis. Além de mostrar potencial antifúngico contra cepas de Candida albicans, atividade que foi atribuída a ação dos compostos das classes de alcaloides, esteróis e flavonoides presentes nas folhas (Fartyal, 2016).

Planta considerada venenosa devido à presença de um glicosídeo cardiotóxico. Ela pode causar dermatite. Já foi relatado que o alto consumo das folhas e seiva podem acarretar em diarreia persistente (Petricevich \& Abarca-Vargas, 2019). Todas as partes são tóxicas, especialmente os frutos, onde após ingestão em alta quantidade pode causar catarse, purgação severa, irritação do trato digestivo, náuseas, salivação excessiva, diarreia e dor (Burrows \& Tyrl, 2013).

\subsection{Catharanthus roseus (L.) Don.}

Conhecida popularmente como "Boa noite" ou "Maria sem-vergonha", Catharanthus roseus é uma erva nativa de Madagascar e possui ampla distribuição em todo o mundo. No Brasil a espécie pode ser encontrada em áreas antrópicas nas diversas regiões (Figura 3) (Flora do Brasil, 2021). Utilizada como ornamental em jardins e projetos paisagísticos, ela possui também amplo potencial medicinal.

Figura 3: Regiões de ocorrência de Catharanthus roseus (L.) Don. no território brasileiro.

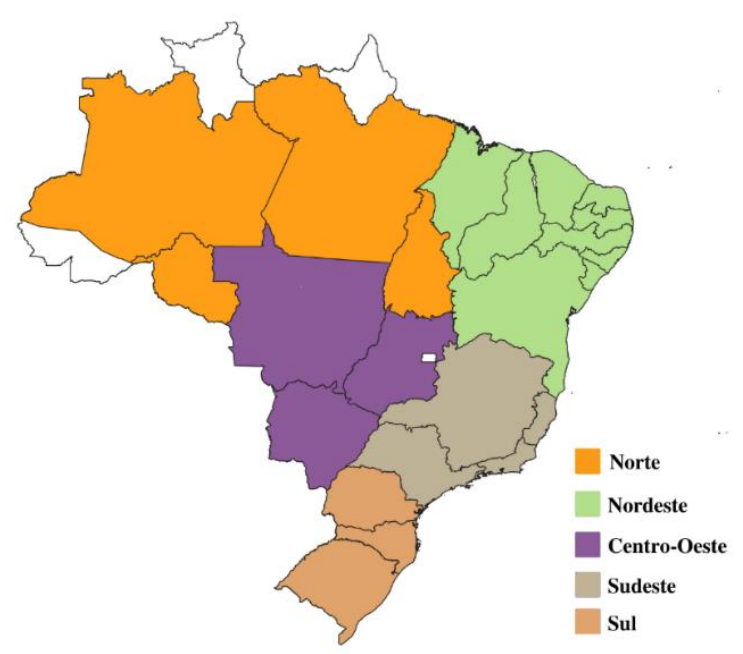

Fonte: Adaptado de Flora do Brasil (2021).

Catharanthus roseus é uma espécie ornamental que se apresenta como uma herbácea perene, com cerca de 30 a $50 \mathrm{~cm}$ de altura. Com folhas elípticas, glabras de cor verde escura brilhante e nervura central pálida. Possui filotaxia oposta cruzada, sendo bastante ramificada. Suas flores são róseas ou brancas com corola com 5 pétalas em forma de lóbulos. Os frutos são do tipo folículo de 2-4 cm e largura de $3 \mathrm{~mm}$, com pequenas e escuras sementes (Lorenzi, 2015; Pham et al., 2020). 
Figura 4: Catharanthus roseus (L.) Don.
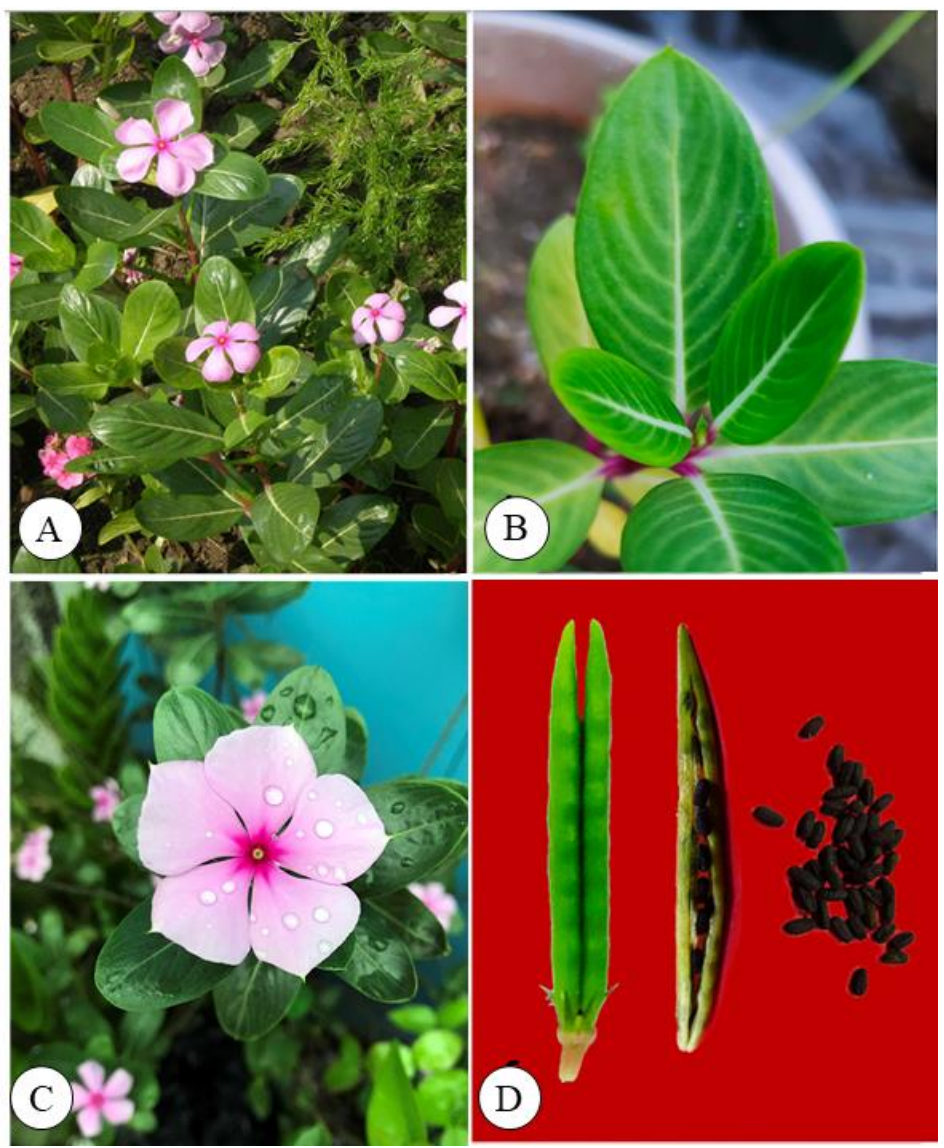

A - Hábito; B - Folhas; C - Flor e D - Fruto maduro e sementes. Fonte: Das et al. (2020).

Historicamente utilizada no tratamento de diversas doenças, no Brasil já foram descritos os usos das folhas de $C$. roseus no controle de hemorragias e escorbuto, como enxaguante bucal para dor de dente, na cicatrização e limpeza de feridas crônicas e no tratamento de diabetes (Nisar et al., 2016; Mishra \& Verma, 2017). O chá preparado utilizando a flor da espécie é utilizado no tratamento de acidente vascular cerebral (AVC), reumatismo, tosse, como calmante e para "recuperar a voz após o derrame" (Arruda, 2012; Vieira et al., 2015).

Catharanthus roseus é utilizada em diferentes países para tratar uma ampla variedade de doenças. No Vietnã e Quênia o extrato das folhas ou da planta inteira é usada como terapias complementares e alternativas no tratamento de diferentes tipos de câncer, incluindo, câncer de garganta, estômago e esôfago. Na Índia as folhas são usadas para tratar picadas de vespas. Na China é utilizada como diurético, adstringente e para tosse. No Havaí, a planta fervida é usada para fazer cataplasma para estancar sangramentos (Nisar et al., 2016; Mishra \& Verma, 2017; Pham et al., 2020).

A espécie possui a capacidade de sintetizar uma grande variedade de compostos que são usados pelo vegetal para realizar funções biológicas, como a defesa de predadores. Os alcaloides é o grupo de compostos mais potencialmente ativo de C. roseus, sendo que já foram registrados mais 400 alcaloides nas diversas partes da espécie. Alcaloides como vimblastina e vincristina estão presentes principalmente nas partes aéreas, enquanto vinceine, vineamine, raubasin, reserpina e catarantina podem ser encontrados nas raízes (Mishra \& Verma, 2017). As raízes de C. roseus contém também os alcalóides ajmalicina e serpentina, compostos com comprovada atividade anti-hipertensiva. $\mathrm{O}$ caule e as raízes da espécie possuem ainda, quantidade considerável de cumarina e quinonas com atividades antibacterianas. Suas flores são ricas em triterpenóides, taninos e alcalóides, compostos com propriedades cicatrizantes de feridas diabéticas (Afolayan \& Sunmonu, 2010). Além destes, $C$. 
roseus possui amplo espectro de compostos fenólicos, incluindo flavonoides, antocianinas, ácido 2,3-di-hidroxibenzóico, fenilpropanóides, quercetina, canferol, malvidina e derivados do ácido cinâmico (Afolayan \& Sunmonu, 2010).

Através da investigação do potencial medicinal de C. roseus, pesquisadores descobriram que a espécie contém alcaloides que, apesar de tóxicos, possuem importante potencial no tratamento do câncer. Os alcalóides vinblastina e vincristina identificados no caule e folhas da espécie possuem potencial de inibição do crescimento de tumores em humanos. Os extratos das folhas e flores de C. roseus, apresentaram efeitos hipoglicêmicos com a redução do açúcar no sangue de ratos. Os extratos das folhas mostraram atividade antibacteriana contra os microrganismos patogênicos: Pseudomonas aeruginosa, Salmonella typhimuruim e Staphylococcus aureus, podendo ser usados como agente profilático no tratamento de diversas enfermidades (Mishra \& Verma, 2017).

Atividades hipolipidímica, antidiarreica, cicatrizantes e hipotensiva também foram registradas dos extratos das folhas de $C$. roseus em animais de laboratório. Os alcaloides vincamina e vindolina encontrados nas folhas mostraram propriedades antiúlceras, onde o composto vincamina apresentou potencial cerebrovasodilatador e neuroprotetor. Suas folhas também mostraram propriedades importantes contra danos gástricos induzidos em ratos. Enquanto extratos das raízes apresentaram potencial antioxidante (Mishra \& Verma, 2017).

Os casos de intoxicação são relatados especialmente nos preparos farmacêuticos antitumorais com os alcaloides da planta. Os alcaloides vimblastina e vincristina podem causar vômitos, náuseas e diarreias quando administrada por vias intravenosas. Vincristina pode causar trombocitopenia e vimblastina pode provocar depressão da medula óssea, leucopenia ou granulocitopenia. Portanto, produtos da espécie é contraindicada durante a gravidez, pois seus alcaloides podem produzir dano ao embrião ou feto (teratogênicos) e também em casos de leucopenia severa (Horto didático, 2021).

Medicamentos como sulfato de vimblastina (Velban) é extraído a partir do alcaloide vimblastina de C. roseus, e é utilizado no tratamento de neoplasias como: doença de Hodgkins e câncer testicular. O sulfato de (Oncovin) derivado de $C$. roseus é utilizado no tratamento de diferentes enfermidades, incluindo a leucemia e linfomas (Figura 5).

Figura 5: Medicamentos que possuem na composição alcaloides (vimblastina e vincristina) derivados de Catharanthus roseus (L.) Don.

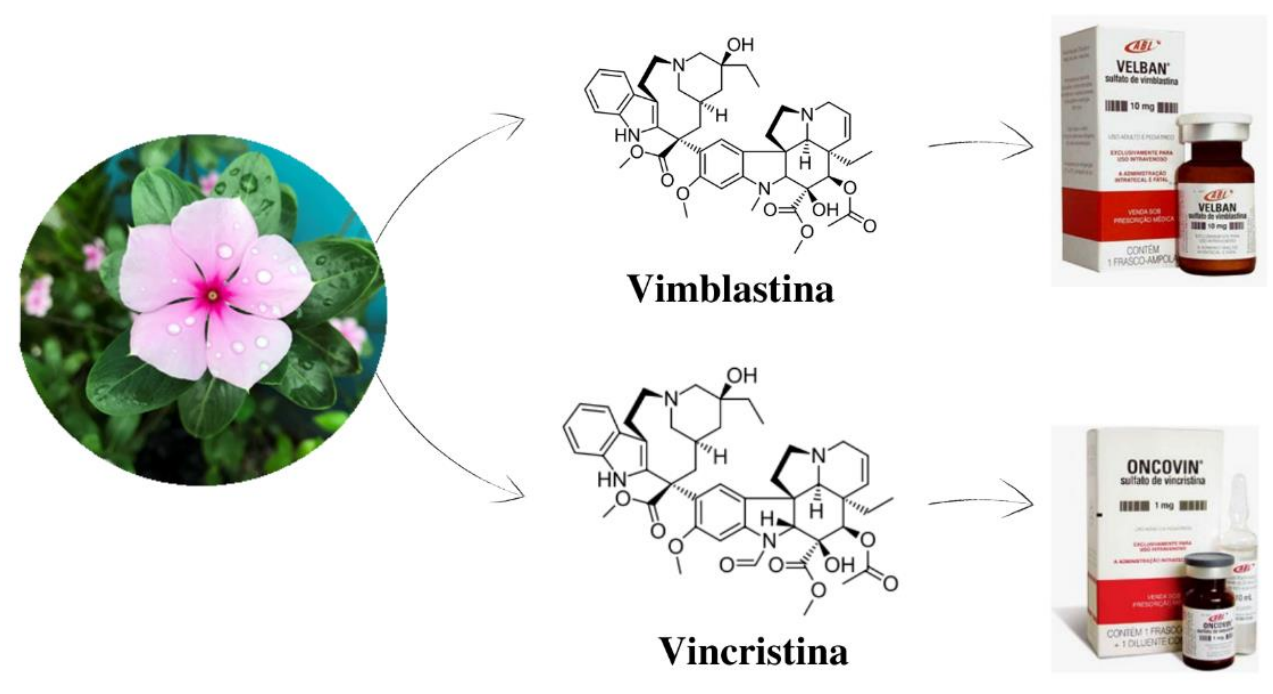

Fonte: Autores (2021). 


\subsection{Nerium oleander $\mathbf{L}$.}

Nerium oleander conhecida no Brasil como "espirradeira" ou "loendro" é uma árvore ornamental (cultivada) com amplo potencial medicinal. Originária da África e Ásia Menor, ela pode ser encontrada em áreas antrópicas das diferentes regiões do Brasil (Flora do Brasil, 2021) (Figura 6).

Figura 6: Regiões de ocorrência de Nerium oleander L. no território brasileiro.

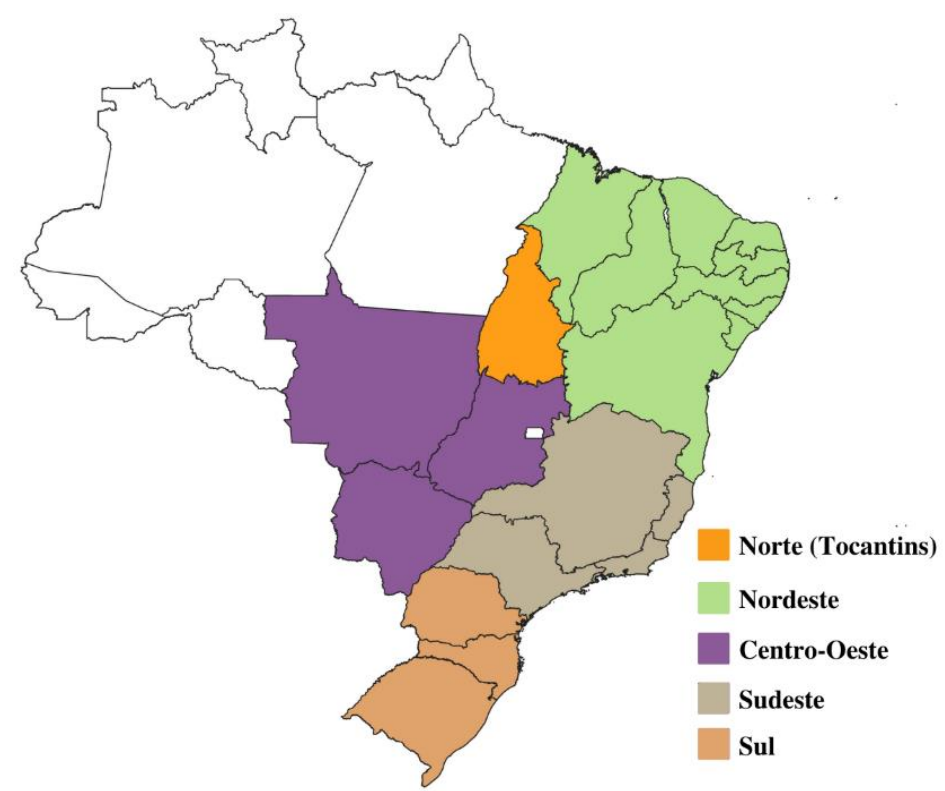

Fonte: Adaptado de Flora do Brasil (2021).

Nerium oleander L. pode atingir de 3 a 5 metros de altura. Suas folhas são em pares ou espirais de três, coriáceas e verde escuras. Possui flores que crescem em cachos no final de cada ramo, com coloração branca, rosa ou vermelha. O fruto é encontrado em formato de cápsula longa e estreita, que se abre para liberar sementes felpudas (Kiran \& Prasad, 2014) (Figura 7). 
Figura 7: Nerium oleander L.

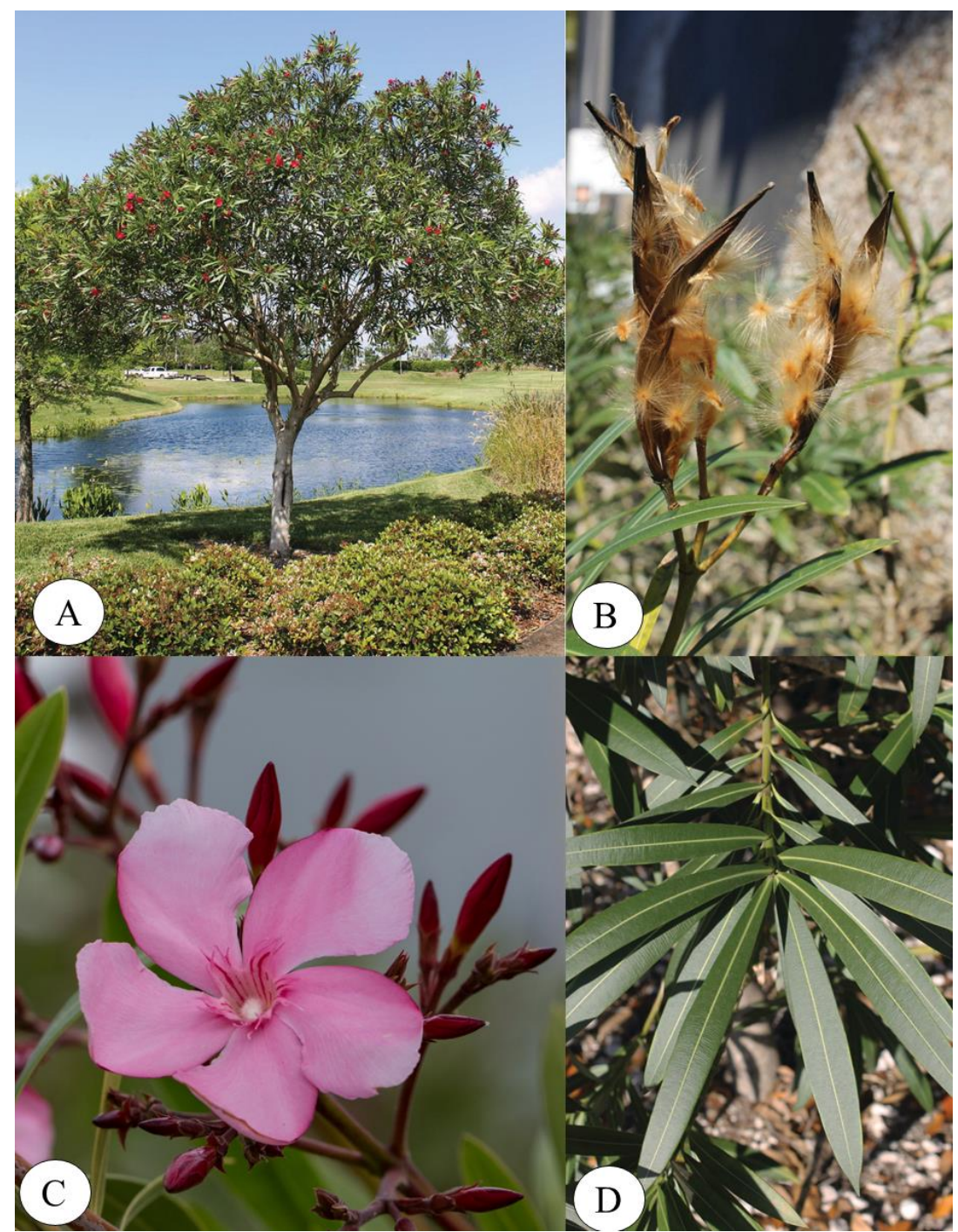

A - Hábito; B - Fruto maduro e sementes; C - Flor e D - folhas. Fonte: Gilman et al. (UF/IFAS Extension) (2019).

Nerium oleander é cultivada em todo o mundo e é uma das plantas ornamentais mais comumente utilizada. A espécie possui também grande potencial medicinal, com registros do seu uso no Brasil como expectorante, tônico para o coração, diurético e emético. Doses muito pequenas de extrato de folhas já foram registradas com uso em picadas de cobra (Ebrahimi $e t$ al., 2018).

O extrato das flores de $N$. oleander possui uso antinociceptivo e cardiotônico. Possuem registros do uso de suas cascas como catártica, febrífuga e febre intermitente. As sementes são utilizadas como purgante em hidropisia e reumatismo. A planta inteira possui propriedades anticâncer, e seu uso neste tratamento foi investigado, a partir do uso popular da planta no tratamento de calos, verrugas, úlceras cancerosas, carcinoma, tumores ulcerativos ou duros (Sinha \& Biswas, 2016; Ebrahimi et al., 2018).

No Egito (Xibin El Kom) N. oleander era utilizada na inalação dos vapores a partir da decocção das raízes no tratamento de dores de cabeça e resfriados, enquanto a decocção das folhas era usada para doenças de pele e paralisia. No deserto do Sinai (Egito), a espécie é amplamente usada na medicina beduína tradicional para tratar cânceres (Hase et al., 2016).

Uma ampla variedade de compostos é encontrada nas diferentes partes de $N$. oleander, incluindo uma grande quantidade de polifenóis que está presente nas folhas, como: ácido cinâmico, epicatequina, catequina e ácido clorogênico. 
Compostos bioativos de diferentes classes são encontrados nas distintas partes do vegetal, como: fenólicos, glicosídeos cardíacos, alcaloides, taninos, flavonoides e terpenóides (Vikas, 2010; Hase et al., 2016).

Quatro cardenolídeos depressores do sistema nervoso central, incluindo neridiginoside, nerizoside, neritaloside e Hodoroside, foram isolados das folhas de $N$. oleander. Dois novos compostos foram isolados do caule da espécie: heptacosano3-enil-5-hidroxihexanoato e 4-oxooctil-2- hidroxiundecanoato. Duas substâncias conhecidas a proceragenina e neridienona A, foram isolados das raízes (Kuete, 2014; Hase et al., 2016).

Nerium oleander é conhecida pelo seu potencial tóxico, em consequência da presença de glicosídeos cardíacos, sendo os mais conhecidos a oleandrina e oleandrigenina. Cardenolideos gentiobiosil oleandrina e odorosídeo também estão presentes. As sementes contêm glicosídeos, incluindo oleandrina, odorosídeos e adigosídeo (Kuete, 2014).

Os extratos das flores e folhas secas de $N$. oleander apresentaram atividade antinociceptiva significativa em contrações abdominais induzidas em camundongos, bem como demonstraram atividade anti-inflamatória contra modelo de edema da pata desses animais. $\mathrm{O}$ extrato das folhas frescas da espécie mostrou efeito depressor do sistema nervoso central (SNC) em camundongos. O óleo essencial das folhas de $N$. oleander apresentaram atividade antitumoral nas linhagens celulares de Carcinoma ascítico de Ehrlich (EAC). Os compostos oleandrina e anvirzel derivados da planta foram capazes de induzir a morte de células tumorais humanas (Vikas, 2010; Sinha \& Biswas, 2016).

A atividade antifúngica do extrato das partes florais da espécie também foi observada contra importantes fungos patogênicos (Fusarium oxysporum, Alternaria alternata, Fusarium solani e Rizoctonia solani). O composto cardenolídeo 12ßhidroxi-5 $\beta$-carda- $8,14,16,20$ (22) - tetraenolídeo, extraído das raízes de $N$. oleander revelou atividades antibacterianas. Os extratos das raízes e folhas da planta mostraram resultados eficazes contra bactérias e fungos. Foram comprovadas também atividades antimicrobianas dos extratos do caule contra Pseudomonas aeruginosa e Bacillus subtilis. O óleo essencial extraído das flores de $N$. oleander mostrou potencial antibacteriano contra Escherichia coli, Pseudomonas aeruginosa e Staphylococcus aureus (Vikas, 2010; Sinha \& Biswas, 2016).

Nerium oleander possui vários compostos tóxicos letais, incluindo nerosídeo e oleandrosídeo e os glicosídeos cardíacos oleandrina e oleandrigenina. Ela pode ser fatal quando ingerida ou preparada incorretamente, portanto nunca deve ser comida cru, como forma de extrato bruto é altamente tóxico. Já foi relatado que os compostos tóxicos da espécie, ao serem ingeridas, paralisam os sistemas cardiovascular e nervoso, após causar fortes náuseas e vômitos (Kuete, 2014; Hase et al., 2016).

Estudos sobre a toxicidade de $N$. oleander, mostraram que cães e humanos, são relativamente sensíveis aos efeitos dos glicosídeos cardíacos e às manifestações clínicas da intoxicação dessas substâncias. Folhas da espécie foram consideradas tóxicas para o gado ao causar morte desses animais em 36 horas após a ingestão. O látex da espécie pode causar irritação e inflamações na pele e olhos. Efeitos gastrointestinais e cardíacos já foram registrados após a ingestão da espécie, incluindo náuseas, vômitos, salivação excessiva, dor abdominal, diarreia e frequência cardíaca irregular (Kuete, 2014).

Venocur é um medicamento que contém em sua composição glicosídeos extraídos das folhas de $N$. oleander, é utilizado no tratamento de sintomas associados a varizes, flebites, úlceras da perna, hemorroidas e outras doenças vasculares. Alzheim Pet é um medicamento homeopático que possui na sua composição $N$. oleander, e é utilizado no tratamento da Síndrome da Disfunção Cognitiva Canina e Disfunção Cognitiva Felina.

\section{Considerações Finais}

Fica evidente que a família Apocynaceae apresenta espécies medicinais de grande importância para as indústrias farmacêuticas, visto que seus representantes são fontes de compostos bioativos. O que indica que são necessários maiores 
estudos sobre o grupo. Bem como os dados aqui apresentados oferecem uma importante base de dados para posteriores estudos.

\section{Referências}

Afolayan, A. J., \& Sunmonu, T. O. (2010). In vivo studies on antidiabetic plants used in South African herbal medicine. Journal of clinical biochemistry and nutrition, 47(2), 98-106.

rruda, A. S. Estudo etnobotânico da Família Asteraceae em Camocim de São Félix, Pernambuco. 2012.97 p. (Dissertações de Mestrado - Biologia Vegetal) Universidade Federal de Pernambuco (UFPE), Recife, Pernambuco, 2012.

BFG. (2015). Growing knowledge: an overview of seed plant diversity in Brazil. Rodriguésia, 66, 1085-1113.

Burrows, G. E., \& Tyrl, R. J. (2013). Toxic plants of north America. 2 ed. New Jersey: John Wiley \& Sons.

Coutinho, T.S., \& Louzada, R. B. (2018). Flora da Usina São José, Igarassu, Pernambuco: Apocynaceae. Rodriguésia, 69(2), 699-714.

Das, A., Sarkar, S., Bhattacharyya, S., \& Gantait, S. (2020). Biotechnological advancements in Catharanthus roseus (L.) G. Don. Applied microbiology and biotechnology, 104(11), 4811-4835.

Di Stasi L. C., \& Hiruma-Lima, C. A. (2002). Gentianales Medicinais. In: Di Stasi L. C.; Hiruma-Lima, C. A. (orgs.) Plantas medicinais na Amazônia e na Mata Atlântica. São Paulo: Editora UNESP, 375-385.

Ebrahimi, F., Nohooji, M. G., \& Miri, S. M., (2018). Agronomic and pharmacological aspects of Nerium oleander: an important medicinal plant. In: The First National Congress and International Fair of Medicinal Plants and Strategies for Persian Medicine that Affect Diabetes. Mashhad, Iran, 9-11.

Fartyal, M. (2016) Allamanda cathartica linn.: Extraction and pharmaceutical evaluation of various extracts of leaves and flowers. International Journal of Current Pharmaceutical Research, 8, 28-32.

Flora do Brasil. (2020). Apocynaceae. Jardim Botânico do Rio de Janeiro. https://reflora.jbrj.gov.br/reflora/listaBrasil/FichaPublicaTaxonUC/Fich aPublicaTaxonUC.do?id=FB33737. Acesso em: 24 abr. 2021.

Ghosh, C. et al. (2019). Allamanda cathartica Linn. Apocynaceae: A mini review. World. International Journal of Herbal Medicine, 7(4), 29-33, 2019.

Grandi, T. S. M. et al. (1989). Plantas medicinais de Minas Gerais, Brasil. Acta Botanica Brasilica, 3(2), 185-224.

Hase, G. J., Deshmukh, K. K., Murade, V., Pokharkar, P. D., Phantanagre, N. D., Hase, D, P., Dichayal, S., \& Gosavi, A. B. (2016). Phytopharmacology of Nerium oleander L. A review. International Journal of Phytopharmacology, 7(2), 0975-9328.

Horto didático. (2021). Horto didático de plantas medicinais do HU/CCS UFSC. Disponível em: https://hortodidatico.ufsc.br/alcachofra/. Acesso em: 26 abr. 2021.

Kiran, C., \& Prasad, D. N. (2014) A review on: Nerium oleander Linn. (Kaner). International Journal of Pharmacognosy and Phytochemical Research, 6(3), 593-597.

Kuete, V. (2014). Physical, hematological, and histopathological signs of toxicity induced by African medicinal plants. Toxicological survey of African medicinal plants., 635-657.

Lopes, R. K., Ritter, M. R., Rates, S. M. K. et al. (2009). Revisão das atividades biológicas e toxicidade das plantas ornamentais mais utilizadas no Rio Grande do Sul, Brasil. Revista Brasileira de Biociências, 7(3), 305-315.

Lorenzi, H. (2015). Plantas para jardim no Brasil: herbáceas, arbustivas e trepadeiras. 2. ed. Nova Odessa, SP: Plantarum.

Mishra, J. N., \& Verma, N. K. (2017). A brief study on Catharanthus roseus: a review. International Journal of Research in Pharmaceutical Sciences, 2(2), $20-23$.

Nisar, A. et al. (2016). An updated review on Catharanthus roseus: phytochemical and pharmacological analysis. Indian Research Journal of Pharmacy and Science, 3(2), 631-653.

Petricevich, V. L., \& Abarca-Vargas, R. (2019). Allamanda cathartica: A Review of the Phytochemistry, Pharmacology, Toxicology, and Biotechnology. Molecules, 2019(24), 1-22.

Pham, H. N. T. et al. (2020). Phytochemicals Derived from Catharanthus roseus and Their Health Benefits. Technologies, 8(4), 80.

Santos, A. C. B., Silva, M. A. P., Santos, M. A. F. \& Leite, T. R. (2013). Levantamento etnobotânico, químico e farmacológico de espécies de Apocynaceae Juss. ocorrentes no Brasil. Revista Brasileira de Plantas Medicinais, 15(3), 442-458.

Scio, E. et al. (2012). Antimicrobial and Antioxidant Activities of Some Plant Extracts. In Phytochemicals as Nutraceuticals: Global Approaches to Their Role in Nutrition and Health. London, UK InTech.

Sinha, S. N. \& Biswas, K. A. (2016). A concise review on Nerium leander L. - An important medicinal plant. Tropical Plant Research, 3 , 408-412.

Vieira, L. S. et al. (2015). Plantas medicinais conhecidas por especialistas locais de uma comunidade rural maranhense. Revista Brasileira de Plantas Medicinais, 17(4), 1061-1068.

Vikas, G. Payal, M. (2010). Phytochemical and pharmacological potential of Nerium oleander: a review. International Journal of Pharmaceutical Sciences and Research (IJPSR), 1(3), 21-27. 